\title{
Stability of coherent synchrotron oscillations with space charge
}

\author{
Oliver Boine-Frankenheim and Oleksandr Chorniy \\ Gesellschaft für Schwerionenforschung mbH, Planckstraße 1, D-64291 Darmstadt, Germany
}

(Received 19 August 2007; published 31 October 2007)

\begin{abstract}
The stability of longitudinal dipole oscillations in Gaussian bunches is studied for different rf wave forms and with nonlinear space charge. In a previous study [O. Boine-Frankenheim and T. Shukla, Phys. Rev. ST Accel. Beams 8, 034201 (2005)], the space charge induced loss of Landau damping and the bunch response to $\mathrm{rf}$ phase modulations was analyzed for elliptic distributions. The present study investigates Landau damping of dipole modes with nonlinear space charge in Gaussian bunches. The stability boundary resulting from a dispersion relation is compared with stability scans performed within a selfconsistent simulation scheme. The results are compared with numerically obtained beam transfer functions.
\end{abstract}

PACS numbers: 29.27.Bd, 29.20.-c, 52.59.Sa, 52.65.Rr

\section{INTRODUCTION}

The longitudinal space charge force plays an important role in the design and in the operation of storage rings or synchrotrons for high current ion beams. Below transition energy, space charge reduces the effective rf voltage seen by the particles in a bunch. This usually requires an increase of the applied rf voltage in order to compensate for the reduction of the bucket area. Space charge affects the frequencies and the damping of coherent bunch modes. This in turn changes the bunch instability thresholds and the corresponding impedance budget.

For an elliptic (Hofmann-Pedersen) bunch distribution the space charge force is directly proportional to the external $\mathrm{rf}$ force. For this distribution the stationary bunch profiles and the incoherent synchrotron frequency distribution with space charge can be obtained analytically $[1,2]$. The magnitude of the rf voltage reduction for an elliptic bunch distribution can be expressed by means of the longitudinal space charge parameter $\Sigma$. For the dipole mode Landau damping is lost when the coherent frequency is outside the band of incoherent synchrotron frequencies. The corresponding threshold space charge parameter has been obtained in Ref. [1] and recently in Ref. [2] for double rf buckets. A double rf system is foreseen in order to increase the bucket area and also the transverse space charge limit in the SchwerIonen-Synchrotron (SIS) heavy ion synchrotron at Gesellschaft für Schwerionenforschung (GSI) [3]. Because of the low injection energy $(11.4 \mathrm{MeV} / \mathrm{u})$ and the demand for highest longitudinal beam quality the intense uranium bunches will be strongly affected by longitudinal space charge. The analysis in Ref. [2] showed that the loss of Landau damping for an elliptic bunch distribution in a double rf bucket occurs already for very small space charge parameters.

The threshold intensities for the loss of Landau damping can be used to predict persistent bunch oscillations. In order to predict stability limits in the presence of narrow band impedances, the concept of effective impedances and related coherent frequency shifts is used (see e.g. [4]). The stability boundary is then defined in the complex effective impedance plane or as a function of the complex coherent frequency shift. The stability boundary for short bunches in single rf buckets with elliptic distributions and space charge was studied analytically in Ref. [5]. With increasing space charge parameter, the stability boundary is shifted along the imaginary impedance axis. Thereby the stability area remains constant. These results were confirmed in part by simulation studies [6].

The observed bunch distributions in heavy ion synchrotrons and storage rings are usually much closer to a Gaussian distribution than to an elliptic distribution. A possible reason lies in the enhanced intrabeam scattering rates in intense heavy ion bunches compared to, e.g., proton bunches with the same space charge parameter. Intrabeam scattering causes a thermalization of initially non-Gaussian bunch distributions. In Gaussian bunches the space charge force is nonlinear, even in the case of a linear external force. Stability boundaries for different bunch distributions in single rf buckets, including also Gaussians, were analyzed, e.g., in Refs. [7,8]. In their stability analysis the spread in synchrotron frequency due to the nonlinear space charge force is treated in the same way as the spread due to the nonlinearity of the external $\mathrm{rf}$ force. However, the space charge induced spread in the synchrotron frequency alone does not cause Landau damping of dipolar type oscillations. This is because the space charge force does not act directly on the bunch center. If, however, the external focusing force is nonlinear, space charge can be effective for Landau damping, in a positive or in a negative way, depending on the polarity of the external nonlinear force. This has been pointed out in Ref. [9] for transverse dipole oscillations. In addition, a dispersion relation accounting explicitly for nonlinear space charge is presented in Ref. [9]. The effect of the space charge induced synchrotron frequency spread on Landau damping in Gaussian bunches has been addressed in Ref. [10] by solving Sacherer's integral equation. It was 
pointed out that space charge alone does not lead to Landau damping. Also the potential importance of mode coupling for the determination of the stability boundaries with space charge was addressed in Ref. [10]. Besides the actual role of mode coupling, an important remaining question is whether nonlinear space charge stabilizes or destabilizes dipolar-type oscillations in Gaussian bunches. In the present study we analyze the stability boundaries for dipolar oscillations with nonlinear space charge in single and in double rf buckets, using different approaches. Simulation studies accounting for self-consistent space charge, external rf fields, and coherent forces play a key role in our analysis. First, in Sec. II the synchrotron equation of motion including space charge and coherent forces is introduced. The conditions for stationary (matched) Gaussian bunch distributions are reviewed in Sec. III. A detailed analysis of the amplitude-dependent synchrotron frequency with nonlinear space charge in single and in double rf buckets is performed in Sec. IV. Afterwards (Sec. V), the dispersion relation for rigid dipole oscillations used in Refs. $[5,10]$ is extended for nonlinear space charge, following closely the procedure described in Ref. [9] for transverse dipole modes. The simulation tool is briefly described in Sec. VI. The stability boundaries obtained from the simulation scans are presented in Sec. VII. The computer beam transfer function (CBTF) is introduced in Sec. VIII and the results are compared with the simulation scans. The summary together with some conclusions are presented in Sec. IX.

\section{COHERENT SYNCHROTRON MOTION: MODEL EQUATION OF MOTION}

Let $\phi$ and $\bar{\phi}$ be the phase coordinates of a particle inside a bunch and of the bunch center. Then the synchrotron equation of motion is

$$
\begin{gathered}
\ddot{\phi}+\omega_{s 0}^{2}\left[V_{\mathrm{rf}}(\phi)+V_{\mathrm{sc}}(\phi-\bar{\phi})\right] \\
=\Omega_{0} \omega_{s 0} Z_{I} \bar{\phi}+\omega_{s 0} Z_{R} \dot{\bar{\phi}}
\end{gathered}
$$

with the synchrotron frequency

$$
\omega_{s 0}^{2}=\frac{q h V_{0}}{R L\left|m^{*}\right|}
$$

the effective mass $m^{*}=-\gamma_{0} m / \eta$, the relativistic parameter $\gamma_{0}$, the slip factor $\eta$, the ring circumference $L$ and radius $R$, the charge $q$, the harmonic number $h$, and the rf voltage amplitude $V_{0}$. The rf voltage form is given through

$$
V_{\mathrm{rf}}(\phi)=[\sin \phi-\alpha \sin (2 \phi)]
$$

with $\alpha=0$ for a stationary single rf wave and $\alpha=1 / 2$ in case of a stationary double rf wave. In the present study, we will only treat bunches stored at a fixed energy. The space charge voltage form is

$$
V_{\mathrm{sc}}(\phi)=-q \beta_{0} c \frac{R X_{s}}{V_{0}} \frac{h^{2}}{R^{2}} \frac{\partial \lambda}{\partial \phi}
$$

with the space charge reactance

$$
X_{s}=\left|\frac{Z_{\|}^{\mathrm{sc}}}{h}\right|=\frac{g_{f}}{2 \epsilon_{0} \beta_{0} c \gamma_{0}^{2}},
$$

the geometry factor $g_{f}$ (see, e.g., Ref. [11]), and the bunch line density

$$
\lambda(\phi)=\frac{d N}{d \phi}=\int_{-\pi}^{\pi} f(\delta, \phi) d \delta .
$$

$f(\delta, \phi)$ is the distribution function in longitudinal phase space with the momentum deviation $\delta=\Delta p / p_{0}$.

The right-hand side (rhs) of Eq. (1) describes the coupling of dipole oscillations to a normalized effective dipole impedance $Z_{\text {eff }}=Z_{R}+i Z_{I}$ (see also [12]). In the absence of this coupling the rigid dipole oscillation frequency is

$$
\Omega_{0}^{2}=\frac{2 \omega_{s 0}^{2}}{N} \int_{0}^{\pi} V_{\mathrm{rf}}(\phi) \lambda^{\prime}(\phi) d \phi .
$$

The effective dipole impedance causes a coherent frequency shift

$$
\Delta \Omega=\frac{i \omega_{s 0}}{2}\left(Z_{R}+i Z_{I}\right)
$$

provided that the shift is sufficiently small $|\Delta \Omega| \ll \Omega_{0}$. In the later Secs. V and VI we will analyze the stability of the dipole oscillations for a given coherent frequency shift $\Delta \Omega$ and a corresponding effective dipole impedance $Z_{\text {eff }}$. It is important to point out that the relationship between the actual longitudinal ring impedance spectrum $Z_{\|}(\omega)$ and the normalized effective dipole impedance can be obtained using different approximations of Sacherer's integral equation (see, e.g., Refs. [5,13]). A detailed analysis of this relationship is not the aim of our present study. Instead we would like to refer to expressions that can be found in the existing literature. In Ref. [13] it is shown that the effective impedance for the dipole mode can be obtained from an expression similar to (see also Ref. [4], page 320)

$$
Z_{R}+i Z_{I}=\frac{q^{2} N \beta_{0} c}{m^{*} \omega_{s 0}^{2} L^{3}} \frac{\sum_{h}\left|\rho\left(\omega_{h}\right)\right|^{2} \frac{Z_{\|}\left(\omega_{h} \pm \Omega\right)}{h}}{\sum_{h}\left|\rho\left(\omega_{h}\right)\right|^{2}}
$$

with the spectral power density of the rigid dipole mode $|\rho(\omega)|^{2}$ at frequencies $h \omega_{0}$. The effective impedance describes the effect of narrow band impedances on the coherent bunch oscillation. Effective impedances for different bunch distributions and bunch modes are obtained, e.g., in Ref. [4]. As an additional remark, we would like to point out that, in the case of rigid dipole oscillations and for small oscillation amplitudes $(\bar{\phi} \ll 1)$, one can expand the oscillating bunch profile as

$$
\lambda(\phi-\bar{\phi}) \approx \lambda(\phi)-\lambda^{\prime}(\phi) \bar{\phi}
$$


with the stationary bunch profile $\lambda(\phi)$ and its derivative $\lambda^{\prime}(\phi)$. Therefore the frequency spectrum of the rigid dipole mode in Eq. (9) can approximated as $\rho\left(\omega_{h}\right) \approx \lambda_{h}^{\prime}=$ $-i h \lambda_{h}$, with the Fourier component $\lambda_{h}$ of the line density at harmonic $h$. However, as mentioned before, in the following sections we will assume given $\Delta \Omega$ and $Z_{\text {eff }}=$ $Z_{R}+i Z_{I}$.

\section{MATCHED BUNCH DISTRIBUTION WITH SPACE CHARGE}

The Hamiltonian corresponding to the equation of motion equation (1) with $Z_{I}=Z_{R}=0$ is

$$
H=\frac{\dot{\phi}^{2}}{2}-\omega_{s 0}^{2} Y(\phi)
$$

with the potential function

$$
Y(\phi)=\int_{0}^{\phi} V(\phi) d \phi
$$

and $V=V_{\mathrm{rf}}+V_{\mathrm{sc}}$. The $\mathrm{rf}$ potential function is

$$
Y_{\mathrm{rf}}(\phi)=1-\cos (\phi)+\alpha \cos (2 \phi)
$$

and the space charge potential function becomes

$$
Y_{\mathrm{sc}}(\phi)=q \beta_{0} c \frac{R X_{s}}{V_{0}} \frac{h^{2}}{R^{2}}\left[\lambda_{0}-\lambda(\phi)\right]
$$

with the line density at the bunch center $\lambda_{0}$. For an elliptic bunch distribution with bunch half-length $\phi_{m}$ and maximum phase velocity $v_{m}=\dot{\phi}_{m}$ in the bunch center, the matched voltage amplitude can be obtained (see Refs. [1,2]) as

$$
\omega_{s 0}^{2}=\frac{v_{m}^{2}}{2 Y_{\mathrm{rf}}\left(\phi_{m}\right)}(1+\Sigma)
$$

with the space charge parameter

$$
\Sigma=\frac{q^{2} h^{3} X_{s} \beta_{0} c \lambda_{0}}{\pi R^{3} m^{*} v_{m}^{2}}
$$

The maximum phase velocity $v_{m}$ is related to the maximum momentum spread $\delta_{m}$ through $v_{m}=$ $-\eta_{0} \beta_{0} c(h / R) \delta_{m}$. The resulting synchrotron frequency shift $\Delta \omega_{s}$ for small $\Sigma$ is

$$
\frac{\Delta \omega_{s}}{\omega_{s 0}}=-\frac{1}{2} \Sigma
$$

For a given space charge parameter, defined for an elliptic distribution, we obtain the rms equivalent Gaussian bunch distribution using the rms momentum spread $\delta_{\text {rms }}$ in the bunch center. For short bunches the relation between these momentum spreads is $\delta_{m} \approx \sqrt{5} \delta_{\text {rms. }}$. The resulting implicit equation for the matched line density of a Gaussian bunch is

$$
\lambda(\phi)=\lambda_{0} \exp \left(-\frac{\omega_{s 0}^{2} Y(\phi)}{2 H_{\mathrm{rms}}}\right)
$$

with

$$
H_{\mathrm{rms}}=\frac{\eta_{0}^{2} h^{2}}{2 R^{2}}\left(\beta_{0} c\right)^{2} \delta_{\mathrm{rms}}^{2}
$$

Equation (18) can be easily solved through a numerical iteration scheme. Example results will be presented in the next section.

\section{INCOHERENT SYNCHROTRON FREQUENCY WITH SPACE CHARGE}

For a given stationary bunch profile $\lambda(\phi)$ in a specific rf bucket form, one can obtain the total potential function $Y(\phi)$. The resulting synchrotron period as a function of the oscillation amplitude $\hat{\phi}$ is

$$
T_{s}(\hat{\phi})=\frac{\sqrt{4}}{\omega_{s 0}} \int_{0}^{\hat{\phi}} \frac{d \phi}{\sqrt{|Y(\phi)-Y(\hat{\phi})|}} .
$$

In the following the resulting synchrotron frequency $\omega_{s}(\hat{\phi})=2 \pi / T_{s}(\hat{\phi})$ will be analyzed for single and double rf buckets.

\section{A. Shielding of the nonlinearity in a single bucket}

In a single rf bucket and for small amplitudes $(\hat{\phi})$, the rf potential can be approximated as

$$
Y_{\mathrm{rf}}(\phi) \approx \frac{\phi^{2}}{2}-\frac{\phi^{4}}{24}
$$

If one further assumes weak space charge $(\Sigma \ll 1)$ and a Gaussian bunch density

$$
\lambda(\phi) \approx \frac{N}{\sqrt{2 \pi} \sigma} \exp \left(-\frac{\phi^{2}}{2 \sigma^{2}}\right),
$$

the space charge potential results as

$$
Y_{\mathrm{sc}}(\phi) \approx-g \Sigma\left(\frac{\phi^{2}}{2}-\frac{\phi^{4}}{8 \sigma^{2}}\right)
$$

with the factor

$$
g=\frac{10}{3} \sqrt{\frac{5}{2 \pi}} \approx 3 .
$$

From the above approximate expressions for $Y_{\mathrm{rf}}$ and $Y_{\mathrm{sc}}$, one can obtain an approximate condition for the cancellation of the rf nonlinearity (the terms proportional to $\phi^{4}$ ) by space charge as

$$
3 g \Sigma=\sigma^{2}, \quad \text { or } \quad \Sigma \approx \frac{\phi_{m}^{2}}{45}
$$

with the bunch length of the equivalent parabolic bunch $\phi_{m}=\sqrt{5} \sigma$. One should recall that for the elliptic distri- 
bution the space charge potential is directly proportional to the rf potential with $Y_{\mathrm{sc}} \approx-\Sigma Y_{\mathrm{rf}}$ (see Ref. [2]). For a Gaussian bunch and for the approximate potential functions $Y_{\mathrm{rf}}$ and $Y_{\mathrm{sc}}$, the calculation of the integral in Eq. (20) yields

$$
\frac{\omega_{s}(\hat{\phi})}{\omega_{s 0}} \approx 1-\frac{1}{2} g \Sigma\left(1-\frac{3 \hat{\phi}^{2}}{8 \sigma^{2}}\right)-\frac{\hat{\phi}^{2}}{16} .
$$

\section{B. Numerical integration: Loss of Landau damping}

Landau damping is lost when the coherent frequency (here the dipole mode) is outside the band of incoherent synchrotron frequencies [1]. Of particular interest is therefore the value $\omega_{s}^{\max }$ and the location $\hat{\phi}$ of the maximum synchrotron frequency. If $\omega_{s}^{\max }$ is below the coherent dipole frequency $\Omega_{0}$, the dipole mode will be undamped. In order to obtain $\omega_{s}^{\max }$ and the corresponding threshold space charge parameter $\Sigma_{\text {th }}$ it is necessary to solve Eq. (20) for larger amplitudes. In this more general case and also for larger space charge parameters, the bunch line density $\lambda(\phi)$ and the synchrotron frequency $\omega_{s}(\hat{\phi})$ must be obtained through numerical integration of Eqs. (18) and (20).

Example results for a single rf bucket are shown in Figs. 1 and 2. Figure 1 shows the line density and the corresponding synchrotron frequency for rms equivalent elliptic and Gaussian bunch distributions with bunch length $\phi_{m}=60^{\circ}$ and space charge parameter $\Sigma=0.1$. For the elliptic distribution the maximum synchrotron frequency at $\hat{\phi}=0$ and $\Sigma=0.1$ is still above the dipole frequency $\Omega_{0}$, indicated as a horizontal line in Fig. 1. For the Gaussian distribution the space charge parameter already exceeds the condition for nonlinearity cancellation at $\Sigma \approx 0.025$.

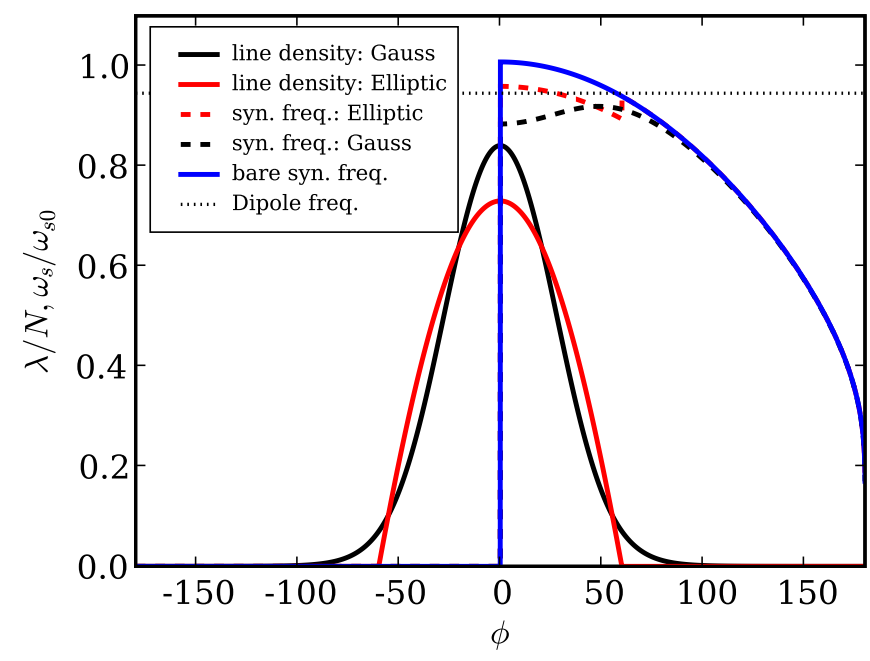

FIG. 1. (Color) Matched line densities for $\Sigma=0.1$ and bunch length $\phi_{m}=60^{\circ}$ in the case of equivalent matched elliptic and Gaussian bunch distributions in a single rf wave. The dashed curves correspond to the respective synchrotron frequencies. The horizontal line represents the dipole frequency.

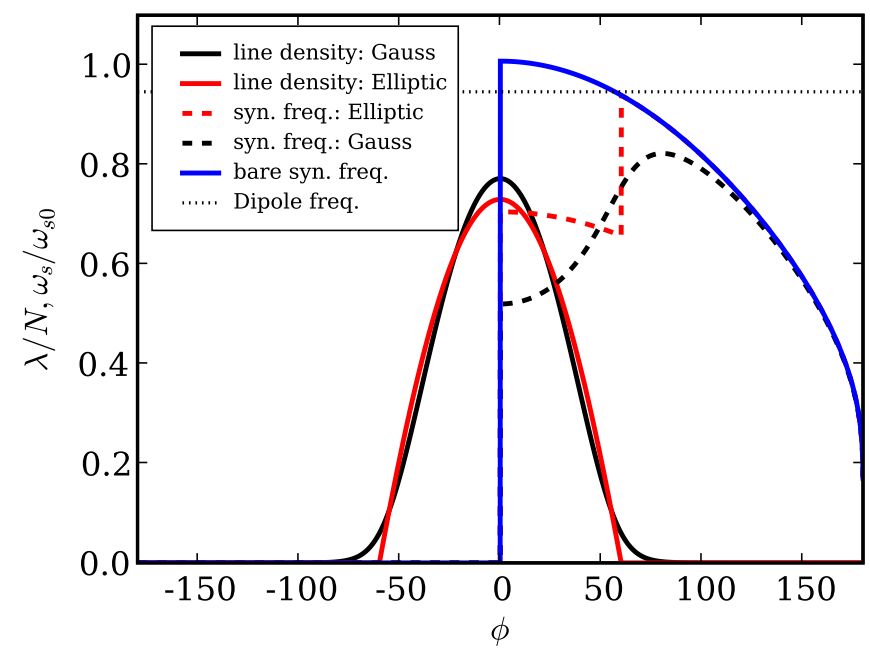

FIG. 2. (Color) Matched line densities for $\Sigma=1$ and bunch length $\phi_{m}=60^{\circ}$ in the case of equivalent matched elliptic and Gaussian bunch distributions in a single rf wave. The dashed curves correspond to the respective synchrotron frequencies. The horizontal line represents the dipole frequency.

The synchrotron frequency exhibits a space charge induced maximum at large amplitudes $\hat{\phi} \gtrsim \phi_{m}$. For $\Sigma=0.1$ this maximum synchrotron frequency $\omega_{s}^{\max }$ is already well below the dipole frequency. For the chosen bunch length of $\phi_{m}=60^{\circ}$ we numerically obtain a threshold space charge parameter of $\Sigma_{\text {th }} \approx 0.04$ for the Gaussian bunch, which is only slightly larger than the value for the cancellation of the rf nonlinearity $(\Sigma \approx 0.025)$. The result for $\Sigma=1$ is shown in Fig. 2. For large space charge parameters the bunch forms obtained from the Gaussian and from the elliptic distribution converge.

The results of the numerical integration of Eqs. (18) and (20) for a double rf bucket are shown in Fig. 3. The space charge parameter is chosen as $\Sigma=1$ and the bunch length is again $\phi_{m}=60^{\circ}$. This bunch length is still below the socalled critical bunch length $\phi_{\text {crit }} \approx 117^{\circ}$. At $\phi_{\text {crit }}$ the synchrotron frequency in a double rf bucket exhibits a maximum $\omega_{s 0}^{\max } \approx 0.78 \omega_{s 0}$ (see Refs. $[14,15]$ ).

In Fig. 3 it can be seen that for $\Sigma=1$ the maximum synchrotron frequency for the elliptic bunch distribution at $\hat{\phi}=\phi_{m}$ is already well below the dipole frequency. In Ref. [2] it has been pointed out that the loss of Landau damping in the case of an elliptic bunch distribution in a double rf bucket occurs at very small space charge parameters $(\Sigma \ll 0.1)$. For the Gaussian distribution and for the chosen bunch length, the maximum synchrotron frequency (at $\phi_{\text {crit }}$ ) still corresponds to $\omega_{s 0}^{\max }>\Omega_{0}$, the maximum synchrotron frequency for $\Sigma=0$.

The above analysis of the incoherent and the coherent synchrotron frequencies indicates that the Gaussian bunch distribution in a double rf bucket is more stable in the presence of space charge, compared to a single rf bucket. In a single rf bucket the maximum synchrotron frequency 


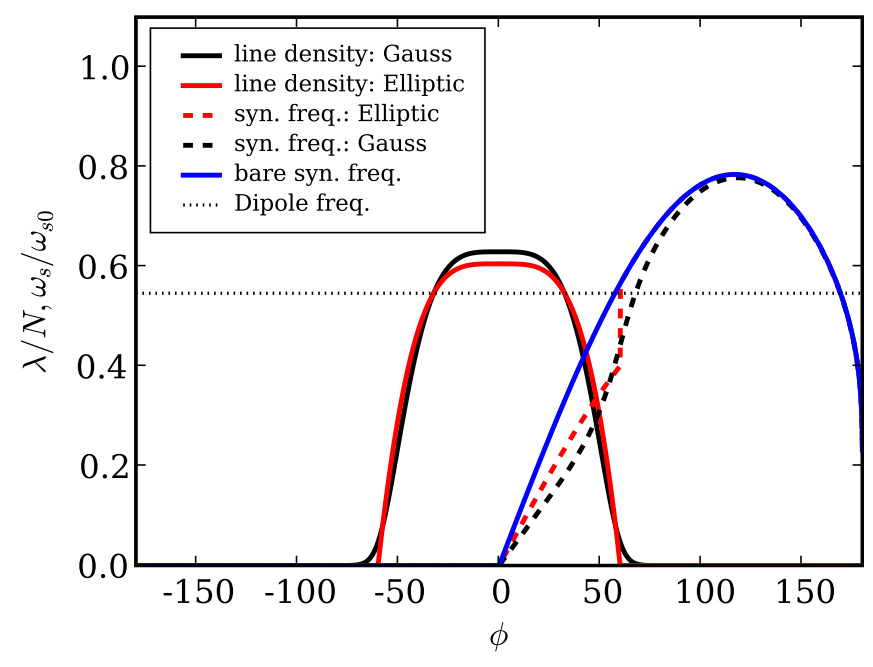

FIG. 3. (Color) Matched line densities for $\Sigma=1$ and bunch length $\phi_{m}=60^{\circ}$ in the case of equivalent matched elliptic and Gaussian bunch distributions in a double rf wave. The dashed curves correspond to the respective synchrotron frequencies. The horizontal line represents the dipole frequency.

is determined by space charge; therefore there is a welldefined threshold space charge parameter $\Sigma_{\text {th }} \ll 1$ for the loss of Landau damping. In a double rf bucket, for bunch lengths $\phi_{m} \gtrsim \phi_{\text {crit }}$ and moderate $\Sigma$, the maximum synchrotron frequency is still determined by the external $\mathrm{rf}$ force. For an elliptic distribution the opposite behavior has been observed in Ref. [2]. In the next section the corresponding stability boundaries will be analyzed.

\section{APPROXIMATE DISPERSION RELATION}

In this section an approximate theoretical approach for the stability analysis of the rigid dipole mode is introduced. The rf nonlinearity is represented through the amplitudedependent incoherent synchrotron frequency $\omega_{s 0}(\hat{\phi})$. The amplitude-dependent frequency shift due to the nonlinear space charge force is $\Delta \omega_{s}(\hat{\phi})$. The coherent forces are represented through the dipole frequency shift $\Delta \Omega$. The approximate longitudinal equation of motion for a test particle is (see also Ref. [9])

$$
\ddot{\phi}-\left(\omega_{s 0}^{2}-2 \omega_{s 0} \Delta \omega_{s}\right) \phi=2 \omega_{s 0}\left(\Delta \Omega-\Delta \omega_{s}\right) \bar{\phi} .
$$

For dipole oscillations the space charge force does not act directly on the bunch center. This is expressed through the driving force $F=2 \omega_{s 0}\left(\Delta \Omega-\Delta \omega_{s}\right) \bar{\phi}$ where the space charge force is subtracted. Assuming small frequency shifts and weak rf nonlinearity, the resulting dispersion relation is (see also Ref. [9])

$$
1=-\pi \int_{0}^{\pi}\left[\Delta \Omega-\Delta \omega_{s}(\hat{\phi})\right] \frac{2 \omega_{s 0} f^{\prime}(\hat{\phi}) \hat{\phi}^{2} d \hat{\phi}}{\Omega^{2}-\omega_{s}^{2}(\hat{\phi})} .
$$

For the elliptic bunch distribution $\Delta \omega_{s}$ is constant and the dispersion relation presented in Ref. [5] is reobtained.
Equation (28) is integrated numerically using the exact synchrotron frequency $\omega_{s}(\hat{\phi})$ for a matched Gaussian bunch distribution. In Fig. 4 the resulting stability boundaries are plotted for different space charge parameters as a function of the normalized coherent frequency shifts $\Delta \Omega / S$ with $S=\omega_{s 0} \sigma^{2} / 16$, the synchrotron frequency spread for a short bunch a single rf bucket. The bunch length is $\phi_{m}=60^{\circ}$. With increasing $\Sigma$ the stability boundary is shifted and the area of stability (the area enclosed between the positive part of the curve and the $\Delta \Omega_{R}$-axis) is reduced in case of a Gaussian distribution. Above $\Sigma \geq 0.1$ the stability boundary extends also to negative values. This would imply that the rigid dipole mode is unstable also in the absence of coherent forces. The self-consistent simulation studies, to be presented in the next section, will show that this is not the case. It is important to point out that for $\Delta \Omega_{R}=0$ Landau damping is lost for relatively small space charge parameters. For $\Sigma=0.05$ one can see in Fig. 4 that at $\Delta \Omega_{R}=0$ the Gaussian bunch is unstable for all $\Delta \Omega_{I}>0$. This loss of Landau damping is due to the shift of the stability area caused by space charge. For an elliptic bunch distribution this shift is given through the incoherent synchrotron frequency shift $\Delta \omega_{s} / \omega_{s 0}=$ $-\Sigma / 2$, for the Gaussian distribution we observe a larger shift $\approx-g \Sigma / 2$, with $g \approx 3$.

Equation (28) can be evaluated also for a double $\mathrm{rf}$ bucket using the numerically obtained synchrotron frequency for a matched bunch distribution. One would expect that the strongly nonlinear synchrotron oscillations in a double rf bucket are beyond the range of applicability of the simple test particle equation (27) and the dispersion relation equation (28). Here we will evaluate Eq. (28) for a double rf bucket in order to compare the obtained stability boundaries with self-consistent simulation results. In Fig. 5

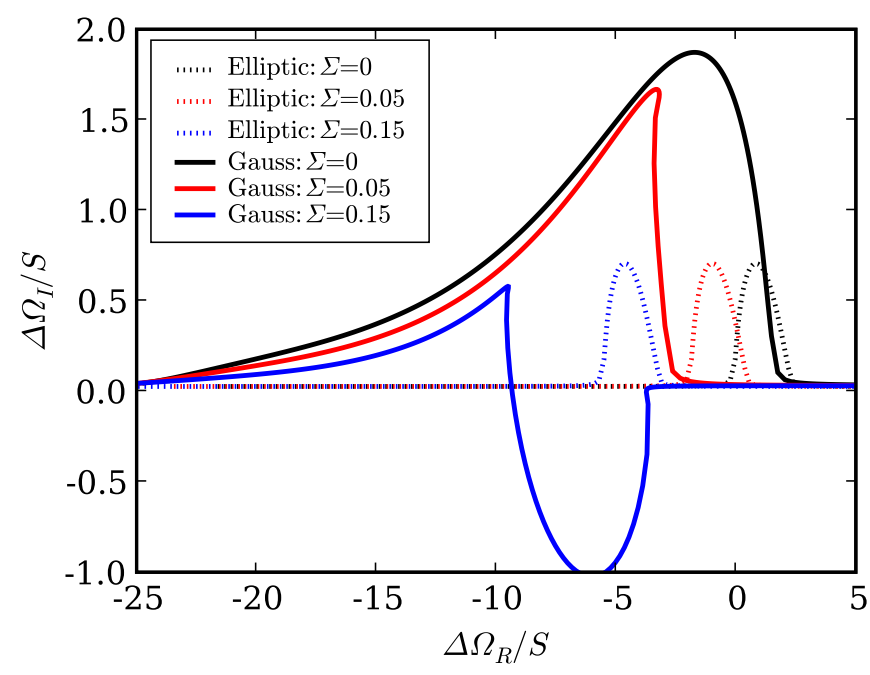

FIG. 4. (Color) Stability boundaries from Eq. (28) as a function of the normalized coherent frequency shifts for different space charge parameters in a single rf wave. The bunch length of the equivalent Gaussian and elliptic distributions is $\phi_{m}=60^{\circ}$. 


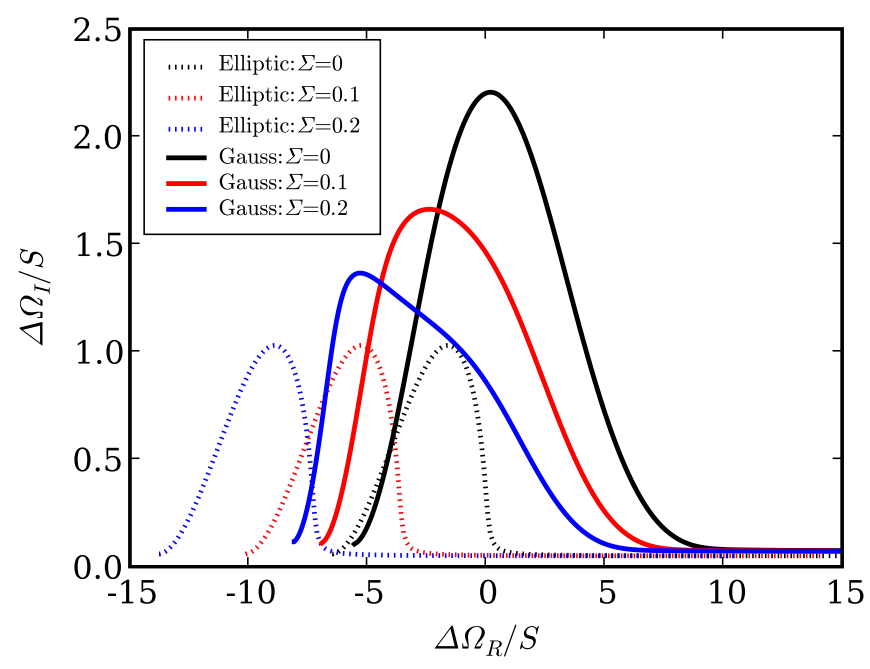

FIG. 5. (Color) Stability boundaries from Eq. (28) as a function of the normalized coherent frequency shifts for different space charge parameters in a double rf wave. The bunch length of the equivalent Gaussian and elliptic distributions is $\phi_{m}=60^{\circ}$.

the stability boundary obtained from Eq. (28) for a double rf bucket is plotted for different space charge parameters and for a bunch length $\phi_{m}=60^{\circ} . S$ is again the frequency spread for a short bunch in a single rf bucket. One can see that for the Gaussian distribution the stability boundary changes with increasing $\Sigma$, but there is no shift and for $\Delta \Omega_{I}=0$ there is always a finite threshold value. This can be understood if we recall (see Fig. 3) that for the chosen bunch length $\left(\phi_{m}=60^{\circ}\right)$ in a double rf bucket the dipole frequency remains inside the band of incoherent synchrotron frequencies even for $\Sigma=1$. This is in contrast to the situation in a single rf bucket or in the case of an elliptic distribution [2] where the dipole frequency for very small $\Sigma$ lies above the incoherent synchrotron frequencies.

\section{SIMULATION SCANS}

In simulation scans Eq. (1) is solved numerically for different effective impedances $\left(Z_{I}, Z_{R}\right)$ and for a fixed initial space charge parameter $\Sigma$ and bunch length $\phi_{m}$. Typically a scan consists of about 100 different simulation runs. The aim of the simulation scans is to resolve the stability boundary in the $\left(Z_{I}, Z_{R}\right)$ plane and to characterize the instability saturation mechanisms.

In order to solve the equation of motion [Eq. (1)] without further approximations, we employ a longitudinal PIC simulation scheme [2] that includes both, the nonlinear external rf fields as well as the self-consistent space charge field calculated using a fast-Fourier transformation on a one-dimensional grid along the $z$ axis with the grid spacing $\Delta z . z=s-s_{0}$ is the relative coordinate of a particle. In order to reduce the effect of artificial noise on the particle dynamics a large number of macroparticles per cell $\Delta z$ is used (typically larger than 1000) together with a quadratic spline interpolation. The number of cells is chosen as $N_{z}=$
512 and the total number of macroparticles is $N_{\text {PIC }}=10^{6}$. The solver is part of the longitudinal beam dynamics code LOBO developed at GSI that incorporates different direct Vlasov solvers as well as the PIC solver used in this work. Both, the Vlasov solvers, operating directly on the distribution function $f(z, v)$, and the PIC solver, operating on macroparticles in $(z, v)$ space, use the same 2D and 1D grids and the same space charge routines. The solvers together with the space charge routine were benchmarked previously against various analytic examples (see [2] and references therein), showing the ability of the solvers to resolve even the finest phase space informations. The code allows one to generate a macroparticle representation from an arbitrary distribution function $f(z, v)$ defined on the 2D grid in $(z, v)$ space. Therefore it is straightforward to load a matched distribution in the PIC solver.

\section{RESULTS OF THE SIMULATION SCANS}

Figure 6 shows the result of a simulation scan for an initial Gaussian bunch distribution with $\Sigma=0$ and bunch length $\phi_{m}=60^{\circ}$ in a single rf bucket. Plotted is the final longitudinal emittance $\epsilon \propto \delta_{m} \phi_{m}$ (divided by the initial value $\left.\epsilon_{0}\right)$ as a function of $\left(Z_{I}, Z_{R}\right)$. The black area in Fig. 6 corresponds to stable initial conditions. One can see that the stability boundary from Eq. (28) (the red curve in Fig. 6) agrees roughly with the area of stability obtained from the simulations. Because of the finite simulation time the small instability growth rates close to the stability boundary cannot be resolved. Therefore a black stripe remains for small $Z_{R}$ outside the stability area predicted by the dispersion relation. The result for a finite space charge parameter with $\Sigma>\Sigma_{\text {th }}$ (here $\Sigma=0.1$ ) is shown in Fig. 7. The reduction of the stability area is quite well

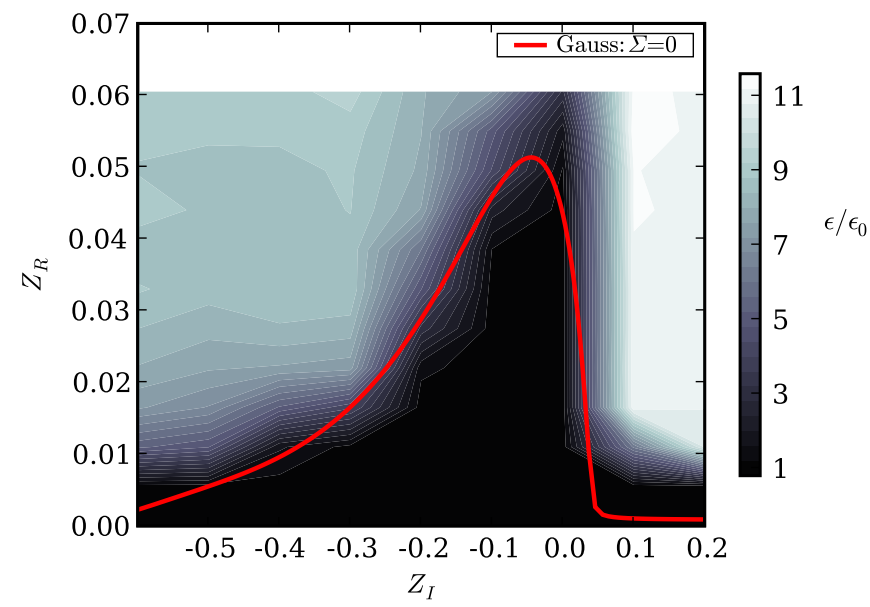

FIG. 6. (Color) Simulation scan of the stability boundary in the normalized impedance plane for a single rf wave and no space charge $(\Sigma=0)$. The bunch length of the initially matched Gaussian distribution is $\phi_{m}=60^{\circ}$. The red curve represents the stability boundary obtained from the dispersion relation equation (28) for $\Sigma=0$. 


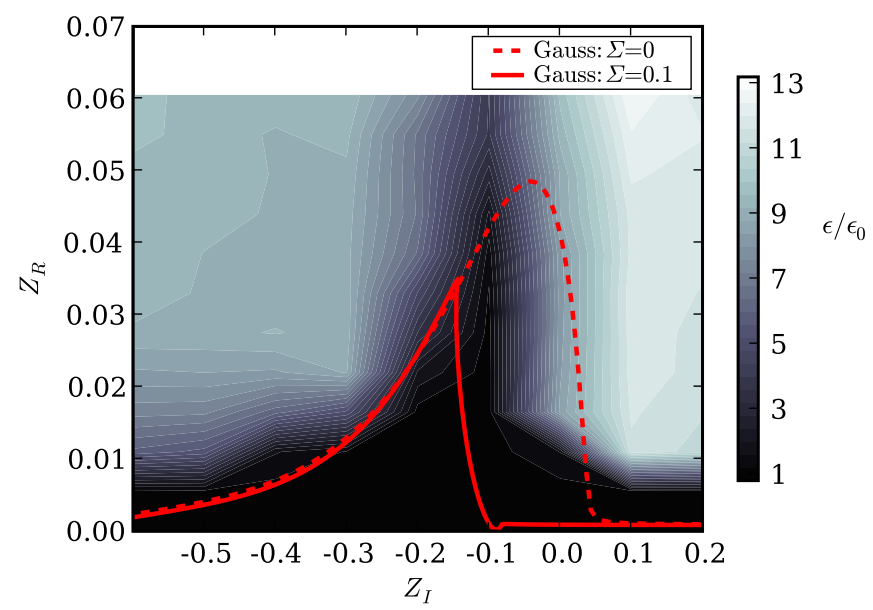

FIG. 7. (Color) Simulation scan of the stability boundary in the normalized impedance plane for a single rf wave and $\Sigma=0.1$. The bunch length of the initially matched Gaussian distribution is $\phi_{m}=60^{\circ}$. The red curves represent the stability boundaries obtained from the dispersion relation equation (28) for $\Sigma=0$ (dashed curve) and $\Sigma=0.1$ (solid curve).

reproduced, but the shift of the stability boundary along the $Z_{I}$ axis is slightly overestimated by the dispersion relation.

A snapshot of the instability evolution for an impedance close to the stability boundary is shown in Fig. 8. One can observe the population of trajectories close to the rf bucket separatrix. In this special case the instability saturation is caused by the formation of a thin longitudinal halo. In other cases, especially for $Z_{I}>0$, the instability causes a more pronounced blowup of the initial bunch distribution. However, the detailed analysis of the complex saturation mechanisms for different impedances and space charge parameters is beyond the present work, focusing on the instability thresholds as a function of the space charge parameter.

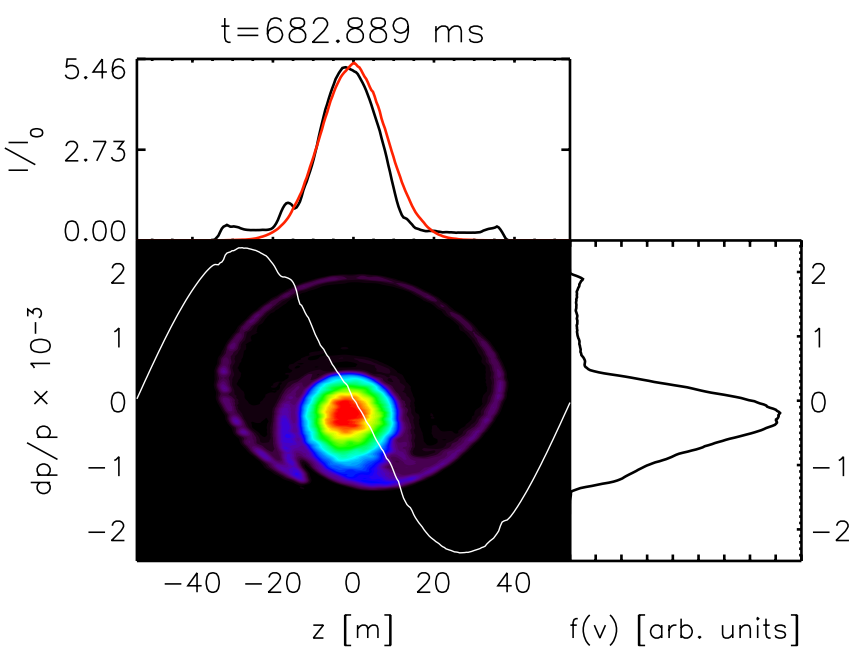

FIG. 8. (Color) Snapshot of the phase space distribution close to the stability boundary for $Z_{I}=-0.2, Z_{R}=0.02, \Sigma=0.1$, and $\phi_{m}=60^{\circ}$.

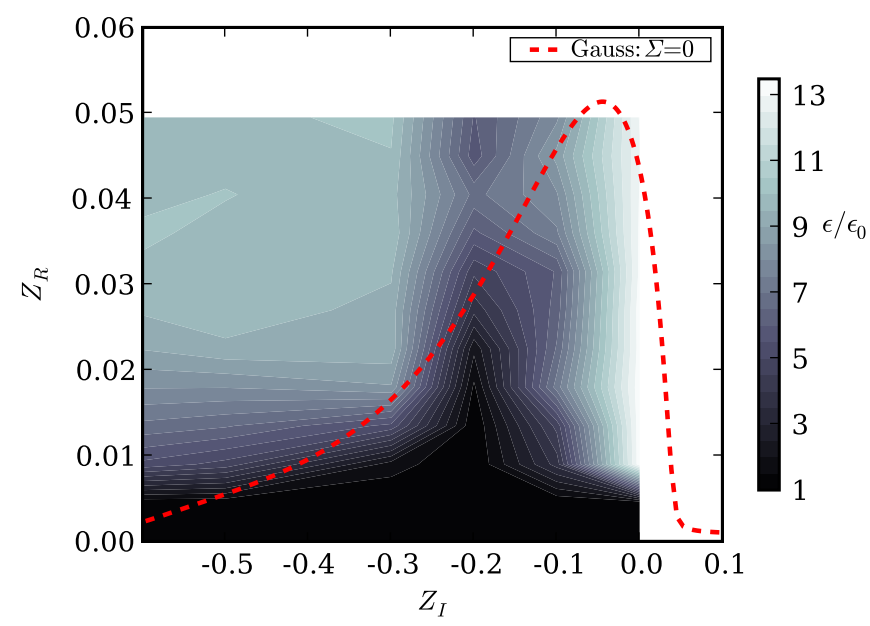

FIG. 9. (Color) Simulation scan of the stability boundary in the normalized impedance plane for a single rf wave and $\Sigma=0.5$. The bunch length of the initially matched Gaussian distribution is $\phi_{m}=60^{\circ}$. The dashed red curve represents the stability boundary obtained from the dispersion relation equation (28) for $\Sigma=0$.

For higher space charge parameters (here $\Sigma=0.5$ ), the stability area obtained from the simulation scan shrinks without being shifted any further (see Fig. 9). For $\Sigma \geqslant 1$ the stability area becomes too small to be observed in our simulation scans. The stability boundary obtained from Eq. (28) for $\Sigma=0.5$ yields negative $Z_{R}$ values. Therefore this result is not shown in Fig. 9. It is important to point out that in all simulation scans we do not observe "antidamping," meaning a finite instability growth rate for $Z_{R} \leq 0$.

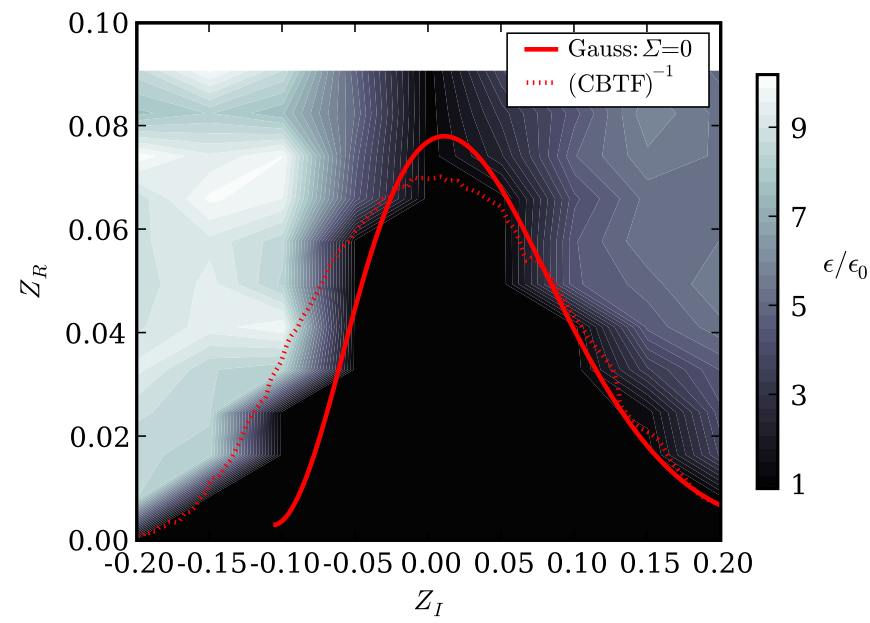

FIG. 10. (Color) Simulation scan of the stability boundary in the normalized impedance plane for a double rf wave and no space charge $(\Sigma=0)$. The bunch length of the initially matched Gaussian distribution is $\phi_{m}=60^{\circ}$. The solid red curve represents the stability boundary obtained from the dispersion relation equation (28) for $\Sigma=0$. The dotted red curve represents the stability boundary obtained from the CBTF (see Sec. VIII). 


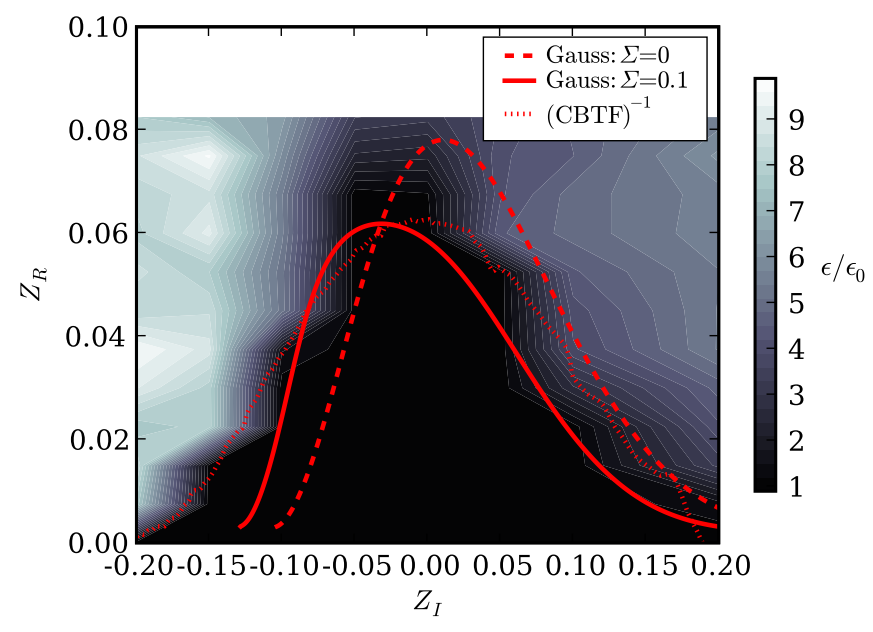

FIG. 11. (Color) Simulation scan of the stability boundary in the normalized impedance plane for a double rf wave and $\Sigma=0.1$. The bunch length of the initially matched Gaussian distribution is $\phi_{m}=60^{\circ}$. The red curves represent the stability boundaries obtained from the dispersion relation equation (28) for $\Sigma=0$ (dashed curve) and $\Sigma=0.1$ (solid curve). The dotted red curve represents the stability boundary obtained from the CBTF (see Sec. VIII) for $\Sigma=0.1$.

The results of simulation scans for double rf buckets are shown in Figs. 10-12. Figure 10 shows the result for an initial Gaussian bunch distribution with $\Sigma=0$ and bunch length $\phi_{m}=60^{\circ}$. For $\Sigma=0$ a relatively good agreement with the stability boundary from the dispersion relation equation (28) is obtained (the solid red curve in Fig. 10). However, for $Z_{I} \approx 0$ a very large threshold $Z_{R}$ is obtained from the simulations, which is not reproduced by the

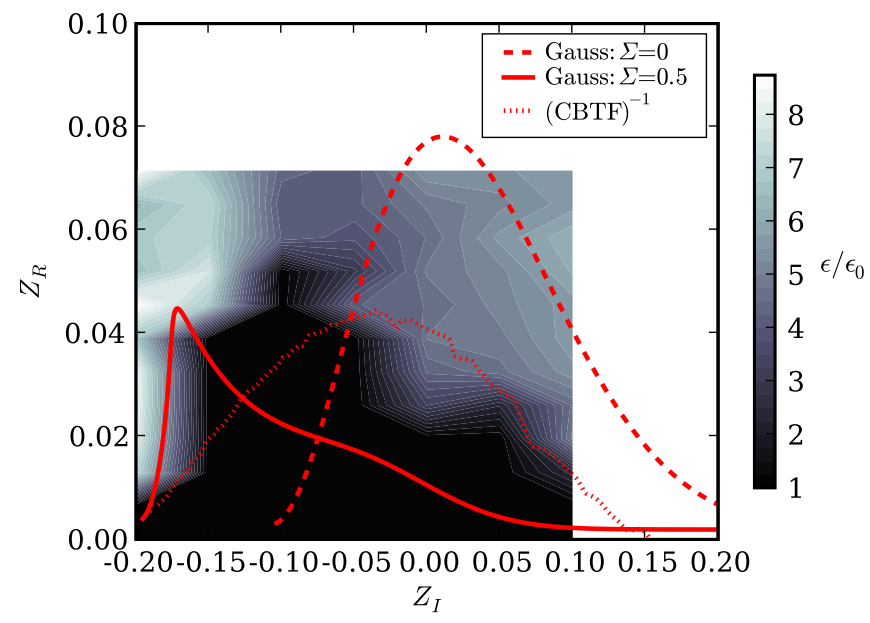

FIG. 12. (Color) Simulation scan of the stability boundary in the normalized impedance plane for a double rf wave and $\Sigma=0.5$. The bunch length of the initially matched Gaussian distribution is $\phi_{m}=60^{\circ}$. The red curves represent the stability boundaries obtained from the dispersion relation equation (28) for $\Sigma=0$ (dashed curve) and $\Sigma=0.5$ (solid curve). The dotted red curve represents the stability boundary obtained from the CBTF (see Sec. VIII) for $\Sigma=0.5$. dispersion relation. For $\Sigma=0.1$ (see Fig. 11) the agreement is still surprisingly good, if we recall the approximations under which the dispersion relation has been obtained. However, for higher space charge parameters (here $\Sigma=0.5$ ) the shift of the stability boundary along the $Z_{I}$ axis predicted by the dispersion relation is too large. In addition the stability area is underestimated.

In summary, the simulation scans provide us with a wealth of information on instability thresholds and saturation mechanisms. For $\Sigma \lesssim 0.1$ the stability boundaries obtained from the simulation and from the dispersion relation equation (28) agree reasonably well. One has to keep in mind that the dispersion relation is based on strong simplifications, e.g., any form of mode coupling is completely neglected by assuming a rigid dipole oscillation. The simulation scans confirm that for Gaussian bunches the stability area in a double rf bucket is less affected by space charge, relative to a single rf bucket. However, quantitatively the stability boundaries obtained from the dispersion relation for large $\Sigma$ disagree with the results of the simulation scans. In order to achieve a better agreement more elaborate theoretical approaches will be required.

\section{VIII. “COMPUTER” BEAM TRANSFER FUNCTION}

The simulation scans are demanding in terms of the required computing resources. A possible alternative to the simulation of stability boundaries is the computer beam transfer function (CBTF). The CBTF should predict, as closely as possible, the results of a real BTF experiment in a storage ring. As in a BTF experiment the rf phase is modulated with the frequency $\Omega$,

$$
V_{\mathrm{rf}}(\phi)=\{\sin [\phi+\hat{\epsilon} \sin (\Omega t)]+\alpha \sin (2 \phi)\},
$$

with the small modulation amplitude $\hat{\epsilon} \ll 1$. Instead of sweeping the modulation frequency $\Omega$ through the synchrotron frequency spectrum, the rf modulation is generated from a bandwidth limited noise spectrum,

$$
\Delta \phi_{\mathrm{rf}}=\hat{\epsilon} \sum_{j} \sin \left(\Omega_{j} t+\chi_{j}\right), \quad \Omega_{j}=\left\{0, \Omega_{\max }\right\},
$$

with a set of randomly chosen frequencies $\Omega_{j}$ and phases $\chi_{j}$. The CBTF is defined as the bunch oscillation spectrum $\bar{\phi}(\Omega)$ divided by the rf modulation spectrum $\Delta \phi_{\mathrm{rf}}(\Omega)$ :

$$
r_{\mathrm{CBTF}}(\Omega)=\frac{\bar{\phi}(\Omega)}{\hat{\epsilon}} \text {. }
$$

The resulting stability boundary $\left(r_{\mathrm{CBTF}}\right)^{-1}$ can be compared with the simulation scans. In addition, one can compare $\left(r_{\mathrm{CBTF}}\right)$ with the $\mathrm{BTF}$ that corresponds to the dispersion relation equation (28)

$$
r^{*}(\Omega)=\frac{r_{0}(\Omega)}{1-D(\Omega)} .
$$

$D(\Omega)$ is the expression on the rhs of the dispersion relation 
equation (28). $r_{0}$ is the BTF in the absence of collective fields $\Delta \omega_{s}=\Delta \Omega=0$.

The CBTF simulations are performed with the LOBO code. Instead of the PIC solver used in the simulation scans, the "noise-free" Vlasov solver is used. This solver has the advantage that smaller rf modulation amplitudes can be used. The only noise source in a Vlasov code is the external perturbation, whereas in a PIC code there is the intrinsic simulation noise in addition (see, e.g., Ref. [16]). In all CBTF simulations external coherent forces are neglected $\left(Z_{R}=Z_{I}=0\right)$ in the equation of motion and only the space charge force is considered. The CBTF obtained for a matched Gaussian bunch (bunch length $\phi_{m}=60^{\circ}$ ) in a single rf bucket with $\Sigma=0$ is shown in Fig. 13. A good agreement with the analytic BTF, $r_{0}$, is obtained (red curves in Fig. 13).

Above the threshold space charge parameter $\Sigma_{\text {th }} \approx 0.04$ (see Sec. IV B) we do not obtain useful results for the
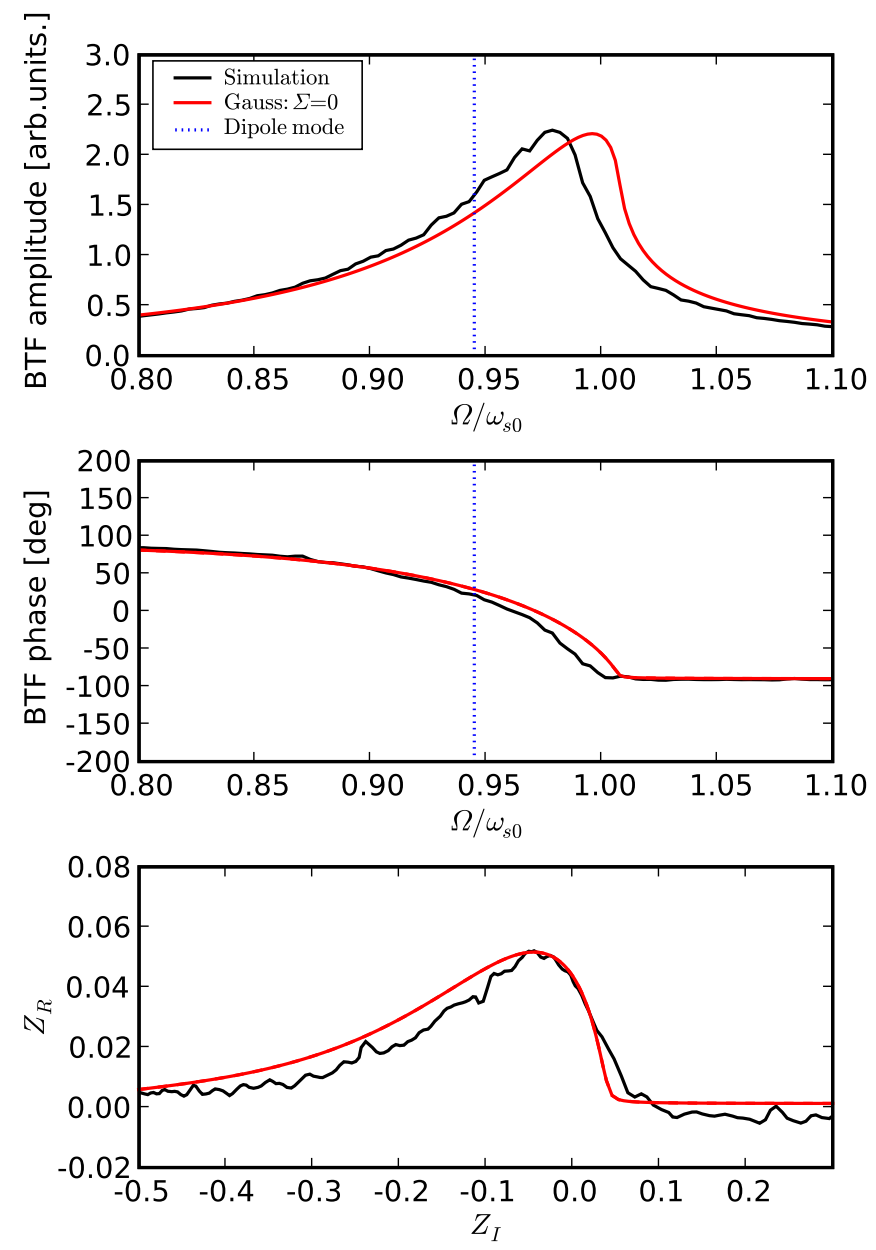

FIG. 13. (Color) CBTF amplitude (top) and phase (middle) as a function of the rf modulation frequency $\Omega$ for a Gaussian bunch in a single rf bucket, bunch length $\phi_{m}=60^{\circ}$, space charge parameter $\Sigma=0$. The resulting stability boundary (bottom) as a function of the effective impedances. The red curves show the BTF and the stability boundary obtained from the dispersion relation.
CBTF. The response amplitude exhibits more than one maximum. The phase and the stability boundary are not well defined. Without Landau damping and in the absence of external damping mechanisms, e.g., electron cooling or feedback systems, the bunch responds like an undamped nonlinear oscillator and a linear response approach is not applicable. Figure 14 shows the CBTF for $\Sigma=0.03$, slightly below $\Sigma_{\text {th }}$. The BTF amplitude and phase from Eq. (32) for $\Sigma=0.03$ (dashed red curve) agree rather well with the simulation result. The stability boundary resulting from the CBTF close to $\Sigma_{\text {th }}$ is already fuzzy.

In a double rf bucket for bunch lengths smaller than $\phi_{\text {crit }}$, Landau damping is effective for realistic space charge parameters (see Sec. IV B). Indeed we obtain smooth results for the CBTF. The stability boundaries from the CBTF for different $\Sigma$ are shown in Figs. 10-12 together with the simulation scans and the results from the
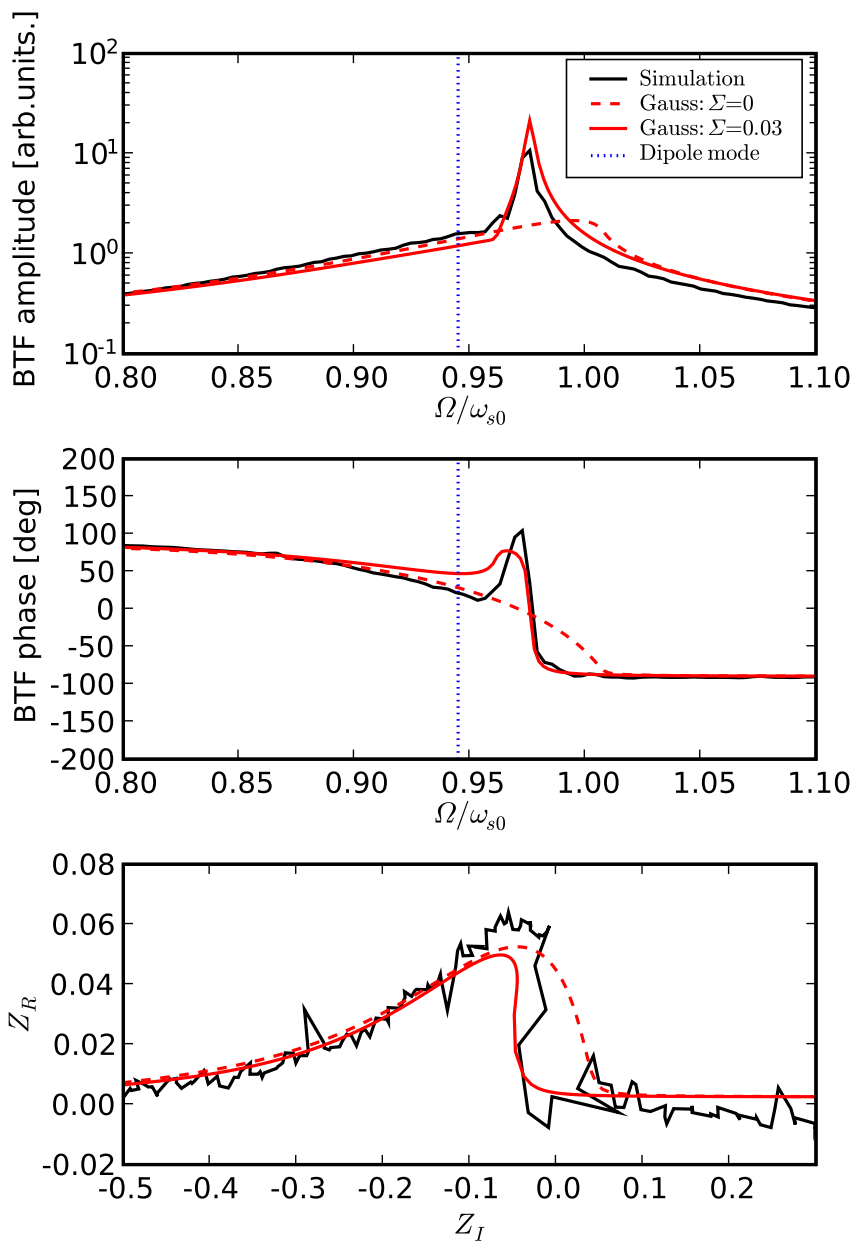

FIG. 14. (Color) CBTF amplitude (top) and phase (middle) as a function of the rf modulation frequency $\Omega$ for a Gaussian bunch in a single $\mathrm{rf}$ bucket, bunch length $\phi_{m}=60^{\circ}$, space charge parameter $\Sigma=0.03$. The resulting stability boundary (bottom) as a function of the effective impedances. The red curves show the BTF and the stability boundary obtained from the dispersion relation. 
dispersion relation. One can see that for $\Sigma=0$ and $\Sigma=$ 0.1 the stability boundary from the CBTF is in accordance with the simulation scans. For higher space charge parameters there is a substantial disagreement (see Fig. 12).

A possible explanation for the disagreement of the inverse CBTF and the stability boundary from simulation scans for higher $\Sigma$ is space charge induced mode coupling. In BTF measurements, one assumes that a single eigenmode is excited, which in the present case of rf phase modulations is the rigid dipole mode. If instead the rigid dipole mode decays due to radial or azimuthal mode coupling [4] then the interpretation of the BTF is more involved and one cannot expect the inverse BTF to agree with the actual stability boundary. In summary, we find that the stability boundary obtained from the CBTF agrees with the simulation scans only for weak space charge.

\section{SUMMARY AND CONCLUSIONS}

The stability of coherent synchrotron oscillations in single and double rf buckets with space charge, below transition and for matched Gaussian bunch distributions has been studied using different numerical approaches. The space charge effect is characterized through the parameter $\Sigma$, which describes the relative rf voltage reduction in the rms equivalent elliptic bunch distribution.

First, the incoherent synchrotron frequency as a function of the amplitude is analyzed. The threshold space charge parameter for the loss of Landau damping in a single rf bucket is obtained numerically. Such a threshold value does not exist in a double rf bucket for short bunches.

Second, an approximate dispersion relation for the rigid dipole mode is introduced and the resulting stability boundary is evaluated numerically including the selfconsistent synchrotron frequency distribution with nonlinear space charge. The resulting stability boundaries for Gaussian and for elliptic bunch distributions are compared. For the elliptic distribution, the stability boundary is shifted with increasing space charge along the imaginary impedance axis, but the stability area remains constant. In the case of a Gaussian bunch in a single rf bucket, the obtained stability area decreases with increasing space charge. The stability boundaries for double rf buckets are much less affected by space charge.

Third, fully self-consistent simulation scans are performed by varying the effective impedance seen by the bunch. The obtained stability boundaries for the single rf bucket show a strong reduction of the stability area with increasing space charge. For weak space charge a sufficient agreement with the stability boundary from the dispersion relation is obtained. Also for the double rf bucket the dispersion relation can sufficiently approximate the stability boundary for small space charge parameters.

Fourth, we compare the beam transfer function obtained from the simulation code (computer beam transfer function: CBTF) with the results from the dispersion relation and with the stability boundaries from the simulation scans. A possible reason for the observed discrepancy between the stability boundaries is mode coupling. We would like to point out that BTF experiments in the SIS with intense ion bunches are presently being performed. The results of these studies and the comparison with the theory described in the present paper will be the topic of a future publication.

In conclusion, we find that below transition nonlinear space charge in Gaussian bunches strongly reduces the stability of the dipole mode in single rf buckets. Gaussian bunches in double rf buckets remain effectively Landau damped with space charge. Future work will focus on Landau damping for higher order modes with space charge. Preliminary results indicate that similar conclusions hold for quadrupole modes in single and in double rf buckets. A final goal of the studies is to identify dangerous undamped bunch modes in order to determine the feedback requirements for intense bunches with space charge.

\section{ACKNOWLEDGMENTS}

The authors acknowledge the support of the European Community Research Infrastructures Action under the FP6 programme: Structuring the European Research Area-Specific Support Action-Design Study (Contract No. 515873-DIRACsecondary-Beams).

[1] A. Hofmann and F. Pedersen, IEEE Trans. Nucl. Sci. 26, 3526 (1979).

[2] O. Boine-Frankenheim and T. Shukla, Phys. Rev. ST Accel. Beams 8, 034201 (2005).

[3] P. Hülsmann, O. Boine-Frankenheim, H. Klingbeil, and G. Schreiber, GSI-Acc-Note-2004-08-001, 2004.

[4] A. W. Chao, Physics of Collective Beam Instabilities in High Energy Accelerators (Wiley, New York, 1993).

[5] K. Y. Ng, Fermilab, Report No. Fermilab-FN-0762-AD, 2005.

[6] O. Boine-Frankenheim, V. Kornilov, and I. Hofmann, Proceedings of EPAC 2006, p. 1886.

[7] F. J. Sacherer, Proceedings of PAC 1973, p. 825.

[8] G. Besnier, Nucl. Instrum. Methods 164, 235 (1979).

[9] D. Moehl, CERN, Report No. CERN/PS 95-08, 1995.

[10] B. Zotter, CERN Report No. SPS/81-19, CERN, Geneva, 1981.

[11] A. Al-khateeb, O. Boine-Frankenheim, I. Hofmann, and G. Rumolo, Phys. Rev. E 63, 026503 (2001).

[12] A. Gerasimov, Phys. Rev. E 49, 2331 (1994).

[13] F. J. Sacherer, Proceedings of PAC 1977, p. 1393.

[14] S. Y. Lee, Accelerator Physics (World Scientific, Singapore, 1999).

[15] E. N. Shaposhnikova, CERN Report No. SL/94-19-RF, 1994.

[16] A. Al-khateeb, O. Boine-Frankenheim, R. Hasse, and I. Hofmann, Phys. Rev. ST Accel. Beams 6, 014205 (2003). 